\title{
Probiotic potential of Escherichia coli ŽP for the gut microbiota of chickens
}

\author{
Irina Maslennikova ${ }^{1, *}$, Julia Gizatullina $^{1}$, Elizaveta Afanasievskaya ${ }^{2}$, Marjanca Starčič \\ Erjavec $^{3}$, and Marina Kuznetsova ${ }^{1}$ \\ ${ }^{1}$ Institute of Ecology and Genetics of Microorganisms, Ural Branch of the Russian Academy of \\ Sciences, Goleva street 13, 614081 Perm, Russia \\ ${ }^{2}$ Perm State Medical University named after E.A. Wagner, str. Petropavlovskaya, 26, Perm, Russia \\ ${ }^{3}$ Department of Biology, Biotechnical Faculty, University of Ljubljana, Jamnikarjeva 101, 1000 \\ Ljubljana, Slovenia
}

\begin{abstract}
The effect of oral administration of Escherichia coli ŽP strain carrying a conjugative plasmid encoding the colicin E7 on the composition of the intestinal chyme microbiota of chickens was studied. It was shown that $E$. coli ŽP was present in the chyme of chickens 6-24 days of age in all experimental groups. In the microbiota of the control group (not administered with the ŽP strain) the Firmicutes phylum, as well as Proteobacteria (E. coli), Actinobacteria (Eubacterium spp.) and, to a lesser extent, Bacteroidetes were found. In the experimental groups the proportion of Firmicutes, represented by the Lactobacillus, Bacillus and Clostridium genera, was higher than in the control group. Our results showed that the use of E. coli ŽP helps to maintain a dynamic state of the microbiota, in all experimental groups, including the group, which was administered an APEC strain.
\end{abstract}

\section{Introduction}

The composition of the microbiota of the chickens is of great importance for their health, especially in the poultry industry. The mortality rate of colibacillosis caused by avian pathogenic Escherichia coli (APEC) can reach 55\% of total losses [1]. Antibiotics have long been used in the poultry industry to treat colibacillosis infections of the respiratory tract, heart and, to a greater extent, the digestive system of birds. The spread of resistant pathogenic strains among avian microbiota called into question the feasibility and effectiveness of antibiotic use and determined the task of finding alternative solutions to the problems $[2,3]$.

Recently, there has been a growing interest in the search for new probiotic drugs, including new cultures of microorganisms based on Escherichia coli, which produce bacteriocins, microcins and can be used in food additives as preventive or therapeutic drugs $[4,5]$. The E. coli ŽP strain created on the basis of Nissle 1917 strain has been recently shown [6] to exhibit killing activity against APEC strains in vitro and in vivo [7].

* Corresponding author: I.Maslennikova1974@gmail.com 
Furthermore, the conjugative method of the colicin E7 DNase activity gene transfer would enable it to act effectively on multiple drug-resistant $E$. coli strains.

The composition of the microbiota in the intestinal tract of chickens depends on the age and the feed of the chicken. It is a highly sensitive indicator system that reacts to any change [8]. Therefore, the study of the probiotic potential of a new strain should include an assessment of its impact on the chicken intestinal microbiome.

In this regard, the purpose of this work was to reveal the effect of per oral administration of E. coli ŽP strain with the conjugative mechanism of colicin E7 delivery on the composition of the chicken intestinal chyme microbiota.

\section{Materials and methods}

\subsection{Strains}

The chloramphenicol- and gentamicin-resistant $\left(\mathrm{Cm}^{\mathrm{r}}, \mathrm{Gm}^{\mathrm{r}}\right)$ potential probiotic E. coli ŽP strain carrying the conjugative plasmid pOX38a with the ColE7 activity gene and the ColE7 immunity gene in the chromosome was used [9]. The ampicillin-resistant (Amp $\left.{ }^{\mathrm{r}}\right)$ APEC strain (RB1) was isolated from organs of chicken with colibacillosis on a poultry farm in the Perm region [7]. Its phylogenetic group (B1) was revealed by quadruplex phylotyping PCR [10]. Furthermore, as shown by PCR using specific primers, the used APEC strain possess following genes: $f l y F$ (haemolysin), fimH (fimbriae), iha (invasion) and blaTEM, blaCTX-M (extended-spectrum $\beta$-lactamase) [7, 10, 11]. The strain RB1 was deposited in the Ex culture collection of the Infrastructural Center Mycosmo (Slovenia) in 2020 as L-5889 strain.

The strains were cultivated in LB media supplemented with appropriate antibiotic (chloramphenicol $50 \mathrm{mg} / \mathrm{L}$ for E. coli ŽP and ampicillin $50 \mathrm{mg} / \mathrm{L}$ for APEC) for $24 \mathrm{~h}$ at $37{ }^{\circ} \mathrm{C}$ with aeration $(180 \mathrm{rpm})$. Next day, the strains were centrifuged, the supernatant removed and the pellet resuspended in $0.9 \% \mathrm{NaCl}$ and administered to the chickens in their drinking water.

\subsection{Experimental groups}

Fifty-five chickens (males and females) of the crossbreed «ROSS-308» (3 days old) were used in experiments. Five experimental groups (ten-twelve animals per group) were formed: control (C) group receiving the main diet and sterile drinking water up to 24 days of age; experimental group 1 - the group receiving the main diet and $10^{8} \mathrm{CFU} / \mathrm{ml}$ of ŽP in the drinking water up to 24 days of age; experimental group 2 - the group receiving the main diet and $10^{10} \mathrm{CFU} / \mathrm{ml}$ of ŽP in the drinking water up to 24 days of age; experimental group 3 - the group receiving the main diet and $10^{8} \mathrm{CFU} / \mathrm{ml}$ of ŽP in the drinking water up to 9 days of age and then sterile drinking water up to 24 days of age; experimental group 4 the group receiving the main diet, $10^{8} \mathrm{CFU} / \mathrm{ml}$ of $\check{Z} \mathrm{P}$ in the drinking water for up to 24 days of age and APEC strain $10^{8} \mathrm{CFU} / \mathrm{ml}$ from 11 days of age up to 24 days of age. Animals received the main diet ad libitum.

All animal protocols were pre-approved by the Ethical Committee of Perm State Medical University named after academician E.A. Wagner (PSMA). All experiments complied with the current laws of Russia as stated in GOST 34088-2017 [12]. 


\subsection{Analysis of microbial diversity}

The intestinal chyme content of 4, 9, 16, 24-day old randomly chosen chickens from each group was used as material for bacteriological and chromatographic analysis.

The bacteriological analysis of microbial intestinal chyme community was performed by direct plating method (colony forming unit count, CFU) on selective solid media: Endo Agar (Russia) for E. coli, Cetrimide Agar (Germany) for Pseudomonas, Ploskireva Agar (Russia) for Proteus, Egg-salt Agar (Russia) for Staphylococcus, Blaurocca medium (Russia) for Bifidobacteria, MRS Agar (Russia) for Lactobacillus, Iron Sulfite Modified Agar №3 (Russia) for Clostridium and Sabouraud Agar №2 (Russia) for Candida. The obtained CFUs were recalculated to $1 \mathrm{~g}$ of the chyme content.

The chromatographic analysis of the microbial intestinal chyme community was performed by determining the polar fatty acid (PLFA) composition as microbial markers using gas chromatography mass spectrometry (GC-MS) (Agilent Technologies, USA) [13, 14]. Lipid components of the chyme content of chickens was extracted by acid methanolysis in $0.4 \mathrm{ml}$ of $1 \mathrm{M} \mathrm{NaCl}$ in methanol for one hour at a temperature of $80^{\circ} \mathrm{C}$. The extract obtained was dried at $80^{\circ} \mathrm{C}$. The dry residue was treated with $20 \mu \mathrm{l}$ of N, O-bis (trimethylsilyl) -trifluoroacetamide for 15 minutes. 1-2 $\mu$ l of the resulting reaction mixture was diluted with hexane and injected into the gas chromatography-mass spectrometer. For quantitative measurements, a hexane solution of tri-decanoic acid deuteromethyl ester was added as an internal standard. An HP-5ms quartz capillary column was used for the analysis. Chromatography was carried out in the temperature programming mode from 130 to $320^{\circ} \mathrm{C}$ at a rate of $5 \mathrm{degree} / \mathrm{min}$. The temperature of the injector was $280{ }^{\circ} \mathrm{C}$, the temperature of the interface was $250{ }^{\circ} \mathrm{C}$. The obtained chromatograms were processed using a standard data processing program and a report was formed on the composition of the microbial community reconstructed by using markers [14].

\subsection{The statistical analysis}

The statistical analysis of the data obtained in triplicates was conducted in MS Excel 2007 and Statistica v. 6.0. The data were expressed as mean values \pm standard error. For data analysed with the $t$-test, the threshold for statistical significance was set at $p$ values $<0.05$. Nonmetric multidimensional scaling (NMS) (difference coefficient is Euclidean distance) was used for analysis of the chromatographic data [15].

\section{Results and discussion}

\subsection{Survival of E. coli ŽP cells in the chick intestine after per oral administration}

As seen from Fig. 1, E. coli ŽP was present in the chicken intestinal chyme throughout the experiment, when the ŽP strain was added to drinking water of chickens of 3-24 days of age (experimental groups 1 and 2), as well as in experimental group 3, where E. coli ŽP was administered in the drinking water only from 3 to 9 days of age and later only sterile water was given for up to 24 days of age. In the control group, the strain was not present (data not shown). In general, the data indicate a good survival rate of E. coli ŽP in the chicken intestines, starting from 3 days of age. 


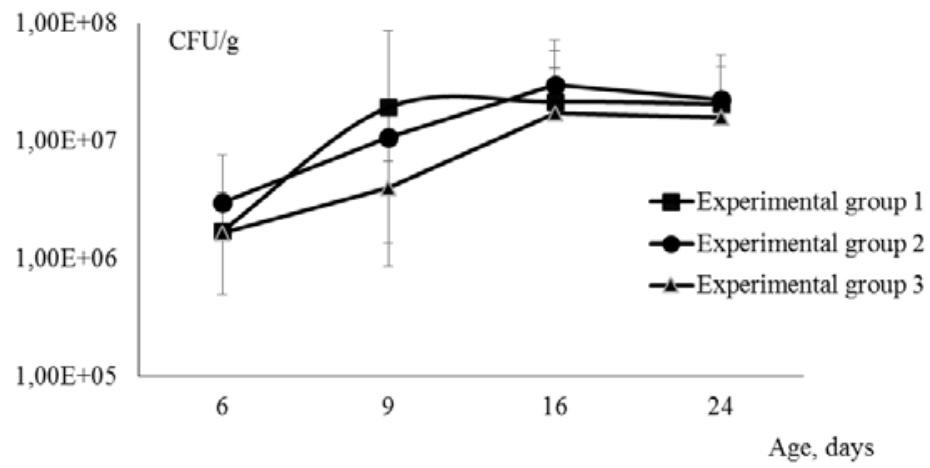

Fig. 1. Colony forming units (CFU) of E. coli ŽP in the intestinal chicken chyme in the experimental groups 1,2 and 3 . The strain was not found in the control group that was not administered ŽP in the drinking water (data not shown).

\subsection{Bacteriological analysis of the intestinal chyme content of chickens}

Bacteriological analysis of the intestinal chyme microbiota of the chickens revealed that the bacteria number of the genus Lactobacillus increased in all experimental groups (1-4), while the proportion of pathogenic and opportunistic microbiota (Proteus, Staphylococcus) slightly decreased in the experimental groups compared to the control group (Fig. 2). However, as this analysis can reveal only a limited number of species, it is rather difficult to determine the advantages or disadvantages of a potential probiotic using this method, especially in the case of modeling an experimental infection with APEC.

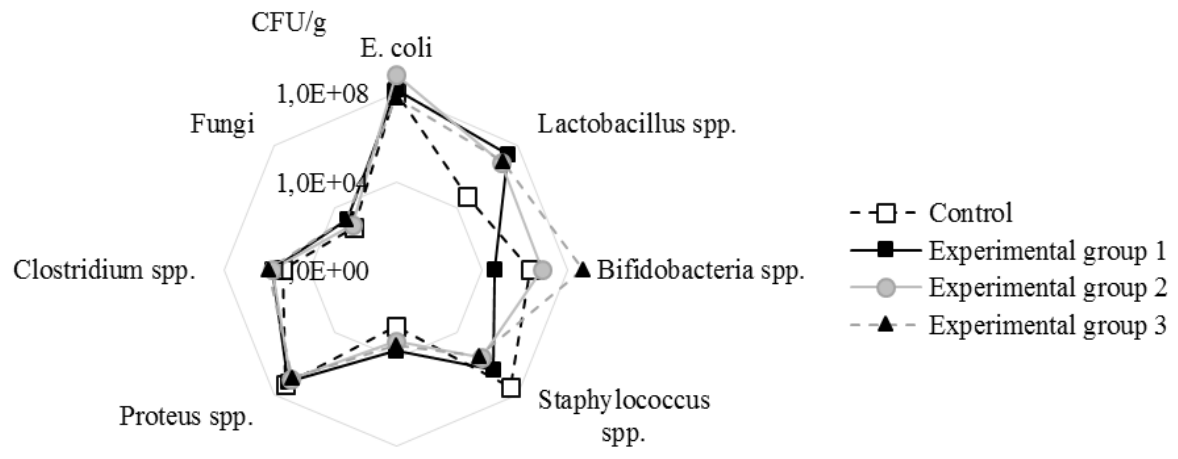

Pseudomonas spp.

Fig. 2. Chyme microbiota composition of chickens at the age of 24 days in the control and experimental groups 1,2 , and 3 as determined by the bacteriological analysis. Data for the experimental group 4 are not shown. 


\subsection{The microbiota composition of chickens based on chromatographic analysis}

The analysis of GC-MS results using the non-metric multidimensional scaling (NMS) method showed that the microbiota composition of the chickens in the control and experimental groups administered the E. coli ŽP strain was different, since their points on the ordination diagram are located in different areas (Fig. 3A). At the same time, a comparison of the groups receiving the probiotic (experimental group 1: drinking water supplemented with $10^{8} \mathrm{CFU} / \mathrm{ml}$ of $\check{Z} \mathrm{P}$ ) and group simulating an APEC infection (experimental group 4: $10^{8} \mathrm{CFU} / \mathrm{ml}$ of ŽP in the drinking water for up to 24 days of age and APEC strain $10^{8} \mathrm{CFU} / \mathrm{ml}$ from 11 days of age up to 24 days of age) revealed that the composition of the microbiota did not vary significantly (Fig. 3B). This may indicate the protective effect of $E$. coli ŽP on the intestinal microbiome of chickens under conditions of APEC infection.
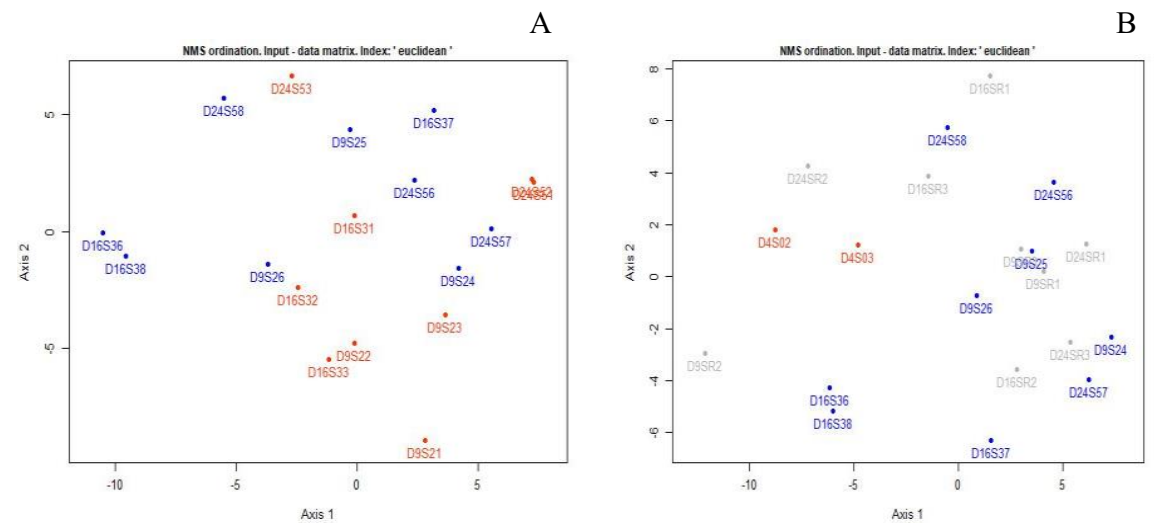

Fig. 3. The microbiota composition of the intestinal chicken chyme revealed by NMS in the control (red dots) and experimental group 1 after per oral administration of E. coli ŽP (blue dots) (A); in the control group before administration of $E$. coli ŽP strain (only 4 days old chickens) (red dots), after the administration of $E$. coli $\breve{Z} \mathrm{P}$ in the experimental group 1 (blue dots) and after the administration of the E. coli ŽP strain in the experimental group 4, which was also administered the APEC strain (gray dots) (B). D4, D9, D16, D24 - samples of intestinal chyme from 4, 9, 16, 24 days old chickens, respectively.

Indeed, the chromatographic analysis of bacterial species in the studied groups revealed that, despite the presence of colibacillosis pathogen (the APEC strain) in drinking water, the potential probiotic E. coli ŽP strain maintained the balance of the microbiota (Fig. 4). So, 24 days old chickens of experimental group 1 and experimental group 4 had similar species profiles.

Initially, bacteria of the phylum Firmicutes (Clostridium spp.) prevailed in the chyme of the chicken intestines (Fig. 4, D4-C), then in the control group by day 24 the microbiota was additionally represented by the phyla Proteobacteria (E. coli), Actinobacteria (Eubacterium spp.) and to a lesser extent Bacteroidetes (Fig. 4, D24-C), which is consistent with the literature [16]. In the experimental groups (Fig 4, D24-1; D24-2; D24-4), the proportion of Firmicutes was higher than in the control group (Fig. 4, D24-C) and was represented by the genera Bacillus and Clostridium. Both bacteria have positive effect on the host health, as it is known that Bacillus acts as a symbiont of the bird intestines, synthesizing antibiotics, enzymes, vitamins, amino acids; and Clostridium degrades dietary fibres in the chicken food [17]. As the administration of the ŽP E. coli strain was correlating with higher levels of bacteria in the genera Bacillus and Clostridium, it can be assumed that the E. coli ŽP strain has a positive effect on chicken health. In the case of an 
administration of a high dosage of probiotic $\left(10^{10} \mathrm{CFU} / \mathrm{ml}\right.$; experimental group 2), it was shown that the overall quantity of bacteria of the intestinal chyme was higher than in other groups due to the phyla Firmicutes and Bacteroidetes. Proteobacteria were represented not only by the E. coli species in this group, but also by other Proteobacteria. We supposed that the most of other E. coli were killed by E. coli ŽP and hence there was "space" for other Proteobacteria (e.g. Helicobacter pylori) to colonize the chicken host.

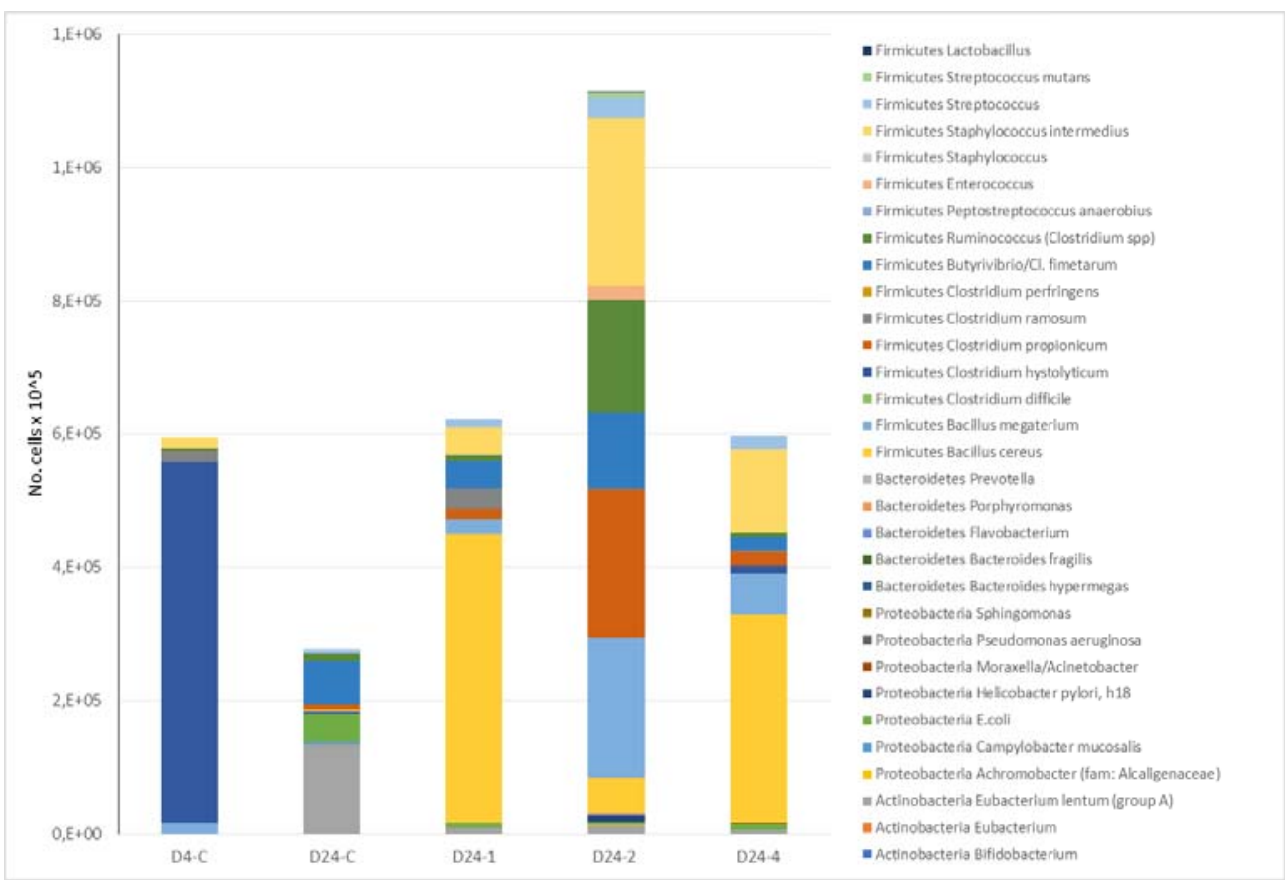

Fig. 4. Intestinal chyme microbiota composition of chickens as determined by chromatographic analysis. Presented data are averaged data from intestinal chyme samples of 4 days old chickens (D4S02; D4S03) and 24 days old chickens (D24S21, D24S22, D24S23 - control group; D24S24-26, D24S27-S29, D24SR1-3 - experimental groups 1, 2, 4, respectively). D4-C and D24-C - control group, 4 and 24 days old chickens, respectively; D24-1, D24-2, D24-4 - experimental group 1, 2, 4, respectively, 24 days old chickens.

It is known that a delayed microbiocenosis formation of the digestive tract of chickens, associated with the sanitary condition of feed, water, and the environment in the conditions of poultry farms, can contribute to the occurrence of gastrointestinal diseases. This work showed that per oral administration of the E. coli ŽP strain affects the composition of the chicken microbiota and, even in the background of administered APEC bacteria, contributes to the maintenance of its dynamic equilibrium.

\section{Acknowledgment}

This research was funded by Government of Perm Krai, grant number No.C-26/792.

\section{References}

1. B.V. Tarakanov, T.A. Nikolicheva, A.I. Manukhina, Agricult. Biology, 42 (2007)

2. H.S. Al-Khalaifah, Poult Sci., 97 (2018) 
3. S. Kumar, C. Chen, N. Indugu, G.O. Werlang, M. Singh, W.K. Kim, H. Thippareddi, PLoS One, 13 (2018)

4. V. Hancock, M. Dahl, P. Klemm, J Med Microbiol., 59 (2010)

5. F. Baquero, V.F. Lanza, M.R. Baquero, R. Del Campo, D.A. Bravo-Vázquez, Front Microbiol., 10 (2019)

6. L. Grozdanov, C. Raasch, J. Schulze, U. Sonnenborn, G. Gottschalk, J. Hacker, U. Dobrindt, J Bacteriol., 186 (2004)

7. M.V. Kuznetsova, I.L. Maslennikova, J.S. Gizatullina, D. Žgur Bertok, M. Starčič Erjavec, Agricult. Biology, 55 (2020)

8. V.I. Fisinin, A.A. Grozina, T.N. Lenkova, V.A. Manukyan, I.A. Egorov, L.A. Il'ina, E.A. Iyldyrym, I.N. Nikonov, V.A. Filippova, G.Y. Laptev, N.I. Novikova, Microbiology (Mikrobiologiya), 85 (2016)

9. M. Starčič Erjavec, Z. Petkovšek, M.V. Kuznetsova, I.L. Maslennikova, D. ŽgurBertok, Plasmid, 82 (2015)

10. O. Clermont, J.K. Christenson, E. Denamur, D.M. Gordon, Environ. Microbiology Reports, 1 (2013)

11. M.V. Kuznetsova, J.S. Gizatullina, L.Y. Nesterova, M. Starčič Erjavec, Microorganisms, 8 (2020)

12. GOST 34088-2017. Guidelines for accommodation and care of laboratory animals. Rules for keeping and care of farm animals (2017)

13. G.A. Osipov, E.S. Turova, FEMS Microbial. Rev., 20 (1997)

14. G.A. Osipov, N.V. Verkhovtseva, Beneficial microbes, 2 (2011)

15. A.B. Novakovskiy, Vestnik instituta biologii Komi nauchnogo centra Ural'skogo otdeleniya RAN, 3 (2016)

16. B.B. Oakley, H.S. Lillehoj, M.H. Kogut, W.K. Kim, J.J. Maurer, A. Pedroso, M.D. Lee, S.R. Collett, T.J. Johnson, N.A. Cox, FEMS Microbiol Lett., 360 (2014)

17. E.A.Yildyrym, L.A. Ilyina, V.A. Filippova, E.P. Gorfunkel, A.V. Dubrovin, N.I. Novikova, D.G. Tyurina, G.Yu. Laptev, Pticevodstvo, 1 (2019) 